\title{
Avoiding Injuries during Inguinal Canal Surgery Case Report
}

\author{
Ashfaq UI Hassan,", Aijaz Malik ${ }^{2}$, Ghulam Hassan ${ }^{3}$ \\ ${ }^{1}$ SKIMS Medical College Srinagar, Kashmir, India \\ ${ }^{2}$ Additional Professor Surgery, SKIMS Soura, Srinagar Kashmir, India \\ ${ }^{3}$ Department of Anatomy, Govt Medical College Srinagar \\ *Correspondence Author: ashhassan@rediffmail.com
}

Copyright (C) 2014 Horizon Research Publishing All rights reserved.

\begin{abstract}
The anatomical localization of Inguinal region is important especially to pediatric surgeons and General Surgeons. A thorough understanding of groin anatomy is essential to successful inguinal hernia treatment. Conservative management of asymptomatic inguinal hernias is usually acceptable and Elective repair of inguinal hernias can be undertaken using a laparoscopic or open approach .Laparoscopic inguinal hernia repair results in less pain and faster recovery, yet requires specialized training and equipment. Without the knowledge of surgical anatomy surgeon can damage many vital structures in this region. The incidence of complications of inguinal hernia surgery is about 10 percent in United states and recent data from India suggests complication rates of order of 20-25 percent in Hospital settings which can increase to about 30-35 percent in District Hospitals will ill equipped theatres and lack of Full Expertise.
\end{abstract}

Keywords Elective Repair, Inguinal Canal, Ilioinguinal, Vas, Genitofemoral, Pampniform

\section{Introduction}

The inguinal canal of the adults is approximately $4 \mathrm{~cm}$ in length. It stretches between the internal or the deep inguinal ring and the external or superficial inguinal ring opening. The inguinal canal contains either the spermatic cord or the round ligament of the uterus. [1]

The canal is bounded superficially by the external oblique aponeurosis. The superior wall is composed of internal oblique muscle, transversus abdominis muscle, and the aponeuroses of these muscles. The inferior wall of the inguinal canal is formed by the inguinal ligament and lacunar ligament. The floor of the inguinal canal is formed by the transversalis fascia and the aponeurosis of the transversus abdominis.

The inferior epigastric vessels serve as a major landmark and form the superolateral border of Hesselbach's triangle.
Hernias occurring within Hesselbach's triangle are considered direct hernias, whereas hernias occurring lateral to the triangle are indirect hernias. [2,3]

From superficial to deep, the structures encountered are Scarpa's fascia after incising the skin and subcutaneous tissue. Deep to Scarpa's layer there is the all important external oblique aponeurosis, which must be carefully incised and spread to identify the cord structures. The medial extension of the external oblique aponeurosis forms the anterior rectus sheath. The iliohypogastric and ilioinguinal nerves, which provide sensation to the skin, penis, and the upper medial thigh, lie deep to the external oblique aponeurosis in the groin region and need to be identified with care. $[4,5,6]$

\section{Objective}

The main objective of the article is to apprise the surgeons of the possible dangers of unintentionally causing damage to structures around the Inguinal canal which can be a source of considerable morbidity as well as mortality .The difference in the open methods of Inginal hernia surgery as well as Laproscopic procedures exist but the relations of inguinal canal remain the same and a surgeon should always be cautious about dissecting in this region. The most effective prophylactic measures necessary for the prevention of complications considered are a thorough knowledge of inguinofemoral anatomy, mature surgical judgment, and meticulous surgical technique such as proper identification of structures, tracing the course of nerves, avoiding low ligation, High ligation in excision of the sac in all hernias, repair of the defect in the plane of its occurrence, and suture of fascia to fascia in the same plane without tension are the basic tenets of inguinal hernia repair which should result in a low incidence of recurrence.

\section{Swellings as Differential Diagnosis}

- Ascites 


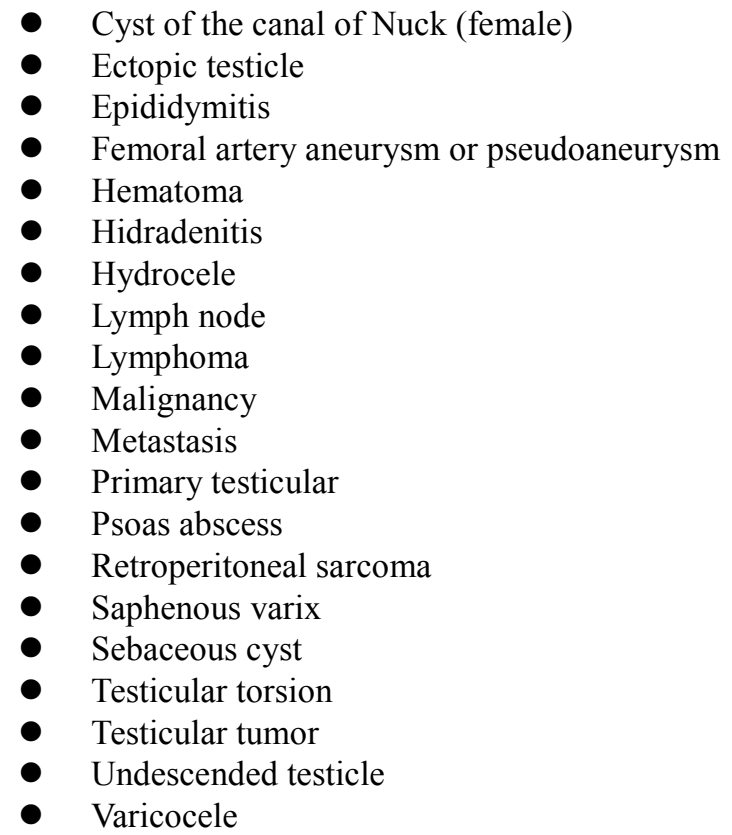

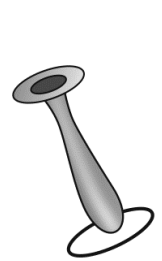

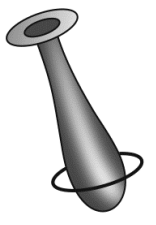

Bubnocele

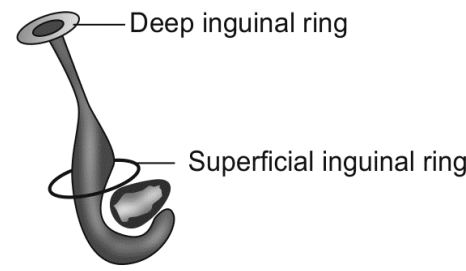

Complete
Figure 1. Different types of Inguinal Hernias

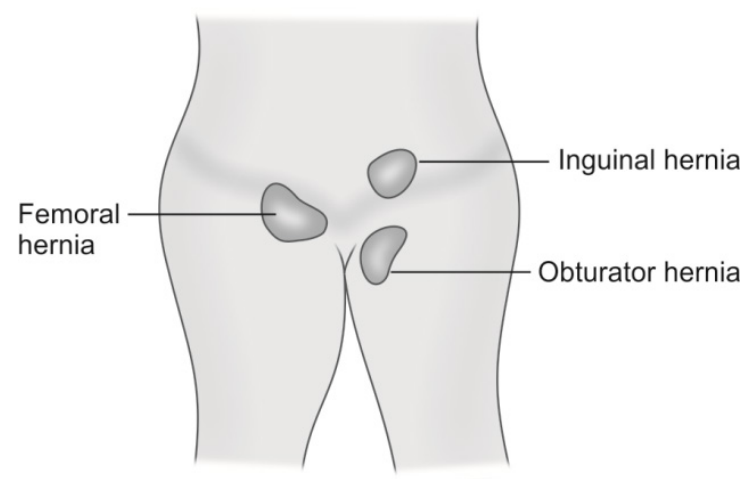

Figure 2. Location point of Inguinal hernia

The factors leading to the development of a hernia are mainly of two categories: congenital and acquired defects. Congenital factors are responsible for the majority of groin hernias. The lack of obliteration of the processus vaginalis is the primary factor leading to the development of an indirect inguinal hernia. Other factors include : $[7,8,9,10]$

- Collagen deficiencies may cause the development of direct inguinal hernias.

- Congenital abnormalities, such as pelvic deformities or exstrophy of the urinary bladder

- low birth weight
- Prematurity

Direct hernias are attributed to exertion mostly likely

- A deficiency of hydroxyproline, the major amino acid component of collagen, in the aponeurosis of patients with groin hernias has been demonstrated.

- Advanced age

- chronic illness

- Heavy lifting

- Intense coughing

- Malnutrition and vitamin deficiency lead to the weakening of strength layers and decreased collagen content, which may lead to hernia formation.

- Several biologic and intracellular factors are closely associated with hernia formation.

- Strain to defecate

- Straining to urinate

Many inborn errors of metabolism can lead to hernia formation. Most common diseases associated are: Ehler Danlos syndrome, Marfan's syndrome, Hunter's syndrome, and Hurler's syndrome can predispose to defects in collagen formation.

Inguinal hernias are at a risk for incarceration and strangulation. Incarceration and strangulation of inguinal hernia may present as a bowel obstruction when the tight hernia defect constricts the lumen of the viscus.

The treatment of hernias, regardless of their type, is surgical repair. Elective repair is performed to alleviate symptoms and to prevent the significant complications of hernias, such as incarceration or strangulation. While the limited data available on the natural history of groin hernias show that these complications are rare, the complications are associated with a high rate of morbidity and mortality when they occur. At the same time, the risks of elective groin hernia repair, even in the patient with a complicated medical history, are exceedingly low. Outcomes of surgical repair are generally excellent .

The major risk with delayed surgical repair is the risk of incarceration and/or strangulation. It is not possible to reliably identify those hernias that are at an increased risk for these complications. It is known that the risk of incarceration of a hernia is greatest soon after the hernia manifests itself. This is likely due to the fact that at the early stage of the hernia, the defect is small and fits tightly around the hernia sac; therefore any contents that fill the sac may quickly become trapped within the hernia. Over time, the hernia defect stretches due to the tissue that enters and leaves the sac with changes in intra-abdominal pressure. In general, the larger the palpable defect on physical examination, the lower the risk of incarceration. Clearly, all risks of tissue loss aside, elective hernia repair is still preferred over emergent repair.

In adult patients, complication rates from open inguinal herniorrhaphy vary. The rate, magnitude, and nature of complications are similar whether the laparoscopic or open approach is used.

Differences occur in the pattern of nerve injury, etiology of visceral injury, and risk of postoperative wound hernia.

Intraoperative complications include injury or transection 
of important structures in spermatic cord, sometimes intense vascular injury producing hemorrhage, cutting or entrapment of nerves, visceral ( bowel or bladder) injury, and systemic complications such as cardiac arrest or death. With the exception of an injury to a nerve, intraoperative complications are usually immediately recognized and repaired.

Injury to the vas is a rare complication of groin hernia surgery in the male patient. Transection of the vas is the most serious form of this injury; this requires urologic consultation and likely immediate reanastomosis in the child or young adult, but may only require ligation of both ends in the older adult patient.

Injury to a nerve, intraoperative complications are usually immediately recognized and repaired.

Postoperative wound complications include infection, hematoma, ecchymosis, and seroma. Complications related to the scrotum and testicles include hematoma, atrophy, sterility, swelling, ecchymosis, and hydrocele. Genitourinary postoperative complications include urinary retention and urinary tract infection.

Recurrence of Hernia especially is seen in old age group, immunocompromised and people with malnourished status and low or poor muscle tone. Recurrence of the hernia in the early postoperative setting is rare. When this does occur, it is often secondary to deep seated infection, undue tension on the repair, or tissue ischemia as a result of poor tissue handling. It may be as a result of Inexperienced Surgeon as well as complicating factors like inadequate fascial strength, poor wound healing, excess trauma, progressive weakening of surrounding tissues, or poor closure technique. The size of the hernia defect is proportional to the risk of hernia recurrence. Larger hernias have an increased rate of recurrence postoperatively.

Nerve injuries are a component usually seen as a result of inguinal hernia repair. $[11,12,13,14]$ The nerves most commonly affected during an open herniorrhaphy are the ilioinguinal nerve and iliohypogastric nerves. During a laparoscopic hernia repair, other nervers that can be damaged include the lateral femoral cutaneous nerve and the genitofemoral nerve. Compression or entrapment of the lateral femoral cutaneous nerve results in meralgia paresthetica - pain and paresthesias in the anterolateral thigh. ${ }^{[15]}$ Rarely, the main trunk of the femoral nerve is injured during an open or laparoscopic inguinal hernia repair.

The ilioinguinal and iliohypogastric nerve arise together from the first lumbar nerve (L1). The ilioinguinal nerve emerges from the lateral border of the psoas major and passes obliquely across the quadratus lumborum. At a point just medial to the anterior superior iliac spine, it crosses the internal oblique muscle to enter the inguinal canal between the internal and external oblique muscles and exits through the superficial inguinal ring. The nerve supplies the skin of the upper and medial thigh. In males, it also supplies the penis and upper scrotum, while supplying the mons pubis and labium majus in females. The iliohypogastric nerve arises from T12-L1 and follows the ilioinguinal nerve. After the iliohypogastric nerve pierces the deep abdominal wall in its downward course, it courses between the internal oblique and transversus abdominis, supplying both. It then branches into a lateral cutaneous branch and an anterior cutaneous branch, which pierces the internal oblique and then external oblique aponeurosis above the superficial inguinal ring.

Persistent neuralgias may be secondary to postoperative inflammatory changes or mild injury to the nerve and surrounding tissues.

The area important laproscopically is referred to as the "Triangle of Doom" . It is a triangular area bordered by the vas deferens medially, gonadal vessels laterally, and peritoneal edge posteriorly, containing the external iliac vessels, the deep circumflex iliac vein, the femoral nerve, and the genital branch of the genitofemoral nerve.Here these structures are at risk of damage and a laproscopic surgeon has to be careful in his manipulation around this region.

The cord structures are formed at the internal ring when the internal spermatic vessels (pampiniform venous plexus and the testicular artery) and the genital branch of the genitofemoral nerve join the vas deferens. The identification of both the vas and the testicular vessels are important to the laparoscopic surgeon as adequate dissection of these structures is essential to assure that a large prosthesis can be placed in the preperitoneal space.

Laproscopically as well when dissecting inferior to the iliopubic tract, care should be taken to prevent injury to the femoral branch of the genitofemoral nerve and the lateral femoral cutaneous nerve, which lie lateral to the spermatic vessels and usually enter the lower extremity just below the iliopubic tract.

Pediatric hernias: The incidence of inguinal hernias in children is more in boys and significantly increased in low-birth-weight and premature infants, secondary to a patent processus vaginalis (PPV) in the latter. The difference in timing of testicular descent results in closure of the left processus vaginalis before the right. Consequently, right-sided hernias are more common. Differential diagnosis of a pediatric groin bulge commonly includes undescended testes, testicular mass, varicoceles, or hydroceles. Unlike in adults, pediatric hernias must be treated emergently, even if they are asymptomatic, to prevent the risk of incarceration. The risk of incarceration has been estimated to be higher with a significantly increased rate of complications.

\subsection{Methods to Prevent Complications of Inguinal Hernia Surgery}

Local anesthesia in primary, inguinal hernia repairs should be considered as a method of choice The incidence of chronic pain is signifi cantly less after identifi cation of all three inguinal nerves than after no identifi cation at all.

Patent processus vaginalis is a risk factor for the development of indirect inguinal hernia.

Mesh repair remains superior to non-mesh inguinal hernia repair over time.

Endoscopic total extraperitoneal repair appears to be 
associated with an increased duration of operation, shorter hospital stay and earlier return to work compared to open inguinal hernia repair Recurrences of inguinal hernia continue to occur up to 10 years after endoscopic hernia repair.

Endoscopic reinforcement of the inguinal posterior wall using a mesh offers good clinical results in athletes with idiopathic groin pain.

\subsection{Treatment Modalities}

Each year about 600,000 hernia-repair operations are performed in the United States. Until recently, however, all were performed as traditional, "open" procedures requiring a large incision in the lower abdomen

Today, the minimally invasive technique of laparoscopic surgery can be used to repair the most common types of hernias. Although both traditional and laparoscopic hernia surgery are performed on an outpatient basis, patients treated laparoscopically seem to experience more rapid healing and far less pain during recovery. Patients can return to normal activity, including work, after only a few days, while recovery from traditional hernia repair can be a three- to five week process.

The laparoscopic approach can be used to repair direct and indirect inguinal (groin) hernias .Postoperativelv, all potential complications, such as hematoma, seroma, chronic pain, and wound infection,. Urinary retention was considerably less in laproscopic procedures.

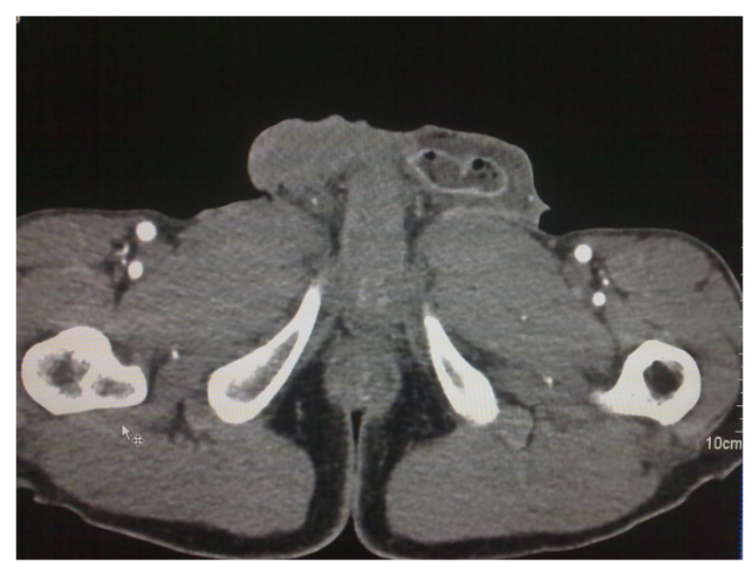

Figure 3. CT Scan Showing Inguinal hernia

\section{Conclusion}

In view of the fact that many patients are operated for the inguinal hernias and in case a surgeon is inexperienced or overenthusiastic, he is bound to damage certain vital structures already mentioned and needs to be extra careful and do a neat dissection and expose the region clearly especially in settings of Primary Health Centers and other centres where full operating facilities are not available. A profound knowledge of Surface and Surgical Anatomy is a fundamental to good surgeon.

\section{REFERENCES}

[1] Skandalakis, J. E., Colborn, G. L., Gray, S. W., et al.: Embryologic and anatomical basis of modern herniorrhaphy: Part II. Anatomy, physiology, and etiology of hernias. Em. Univ. J. Med., 6:177, 1992.

[2] Nyhus, L. M., Klein, M. S., and Rogers, F. B.: Inguinal hernia. Curr. Probl. Surg., 6:401, 1991.

[3] Annibali, R. G.: Surgical anatomy of the inguinal region and lower abdominal wall from the laparoscopic perspective. In Nyhus, L. M., and Condon, R. E. (Eds.): Hernia, 4th ed. Philadelphia, J. B. Lippincott, 1995.

[4] Arregui ME, Davis CJ, Yucel O, Nagan RF. Laparoscopic mesh repair of inguinal hernia using a preperitoneal approach: a preliminary report. Surg Laparosc Endosc 1992;2(1):53-58

[5] Condon RE, Carilli S. The Biology and Anatomy of Inguinofemoral Hernia. Semin Laparosc Surg 1994;1(2):75-85

[6] O'Malley KJ, Monkhouse WS, Qureshi MA, Bouchier-Hayes DJ. Anatomy of the peritoneal aspect of the deep inguinal ring: implications for laparoscopic inguinal herniorrhaphy. Clin Anat 1997;10(5):313-317

[7] Bradburn, N. C., and Schreiner, R. L.: Neonatal seizures. In Schreiner, R. L., and Bradburn, N. C. (Eds.): Care of the Newborn, 2nd ed. New York, Raven Press, 1988, p. 153.

[8] Breslow, N., Churchill, G., et al.: Prognosis for Wilms' tumor patients with nonmetastatic disease at diagnosis: Results of the 2nd national Wilms' tumor study. J. Clin. Oncol., 3:521, 1985.

[9] Brodeur, G. M., and Fong, C. T.: Molecular biology and genetics of human neuroblastoma. Cancer Genet. Cytogenet., 41:153, 1989.

[10] Brynskov, J., Freund, L., Rasmussen, S. N., Lauritzen, K., et al.: A placebo-controlled, double-blind, randomized trial of cyclosporine therapy in active Crohn's disease. N. Engl. J. Med., 321:845, 1989.

[11] Harms, B. A., DeHaas, D. R., and Starling, J. R.: Diagnosis and management of genitofemoral neuralgia. Arch. Surg., $119: 339,1984$

[12] Starling, J. R., Harms, B. A., Schroeder, M. E., and Eichman, P. L.: Diagnosis and treatment of genitofemoral and ilioinguinal entrapment neuralgia. Surgery, 102:581, 1987.

[13] Starling, J. R., and Harms, B. A.: Diagnosis and treatment of ilioinguinal genitofemoral neuralgia. World J. Surg., 13:586, 1989.

[14] Laha, R. K., Rao, S., Pedgeon, C. N., and Dujovny, M.: Genitofemoral neuralgia. Surg. Neurol., 8:280, 1977. 